\title{
RINGO2: an EMCCD-based polarimeter for GRB followup
}

\author{
I. A. Steele ${ }^{a}$, S. D. Bates ${ }^{a}$, C. Guidorzi ${ }^{b}$, C. J. Mottram ${ }^{a}$, C. G. Mundell ${ }^{a}$, R. J. Smith ${ }^{a}$ \\ ${ }^{a}$ Astrophysics Research Institute, Liverpool JMU, Twelve Quays House, Egerton Wharf, \\ Birkenhead CH41 1LD, UK; \\ ${ }^{b}$ Physics Department, University of Ferrara, Via Saragat 1, 44122 Ferrara, Italy
}

\begin{abstract}
We describe the design and construction of a new novel optical polarimeter (RINGO2) for the Liverpool Telescope. The instrument is designed for rapid ( $<3$ minute) followup observations of Gamma Ray Bursts in order to measure the early time polarization and time evolution on timescales of $\sim 1-10000$ seconds. By using a fast rotating Polaroid whose rotation is synchronized to control the readout of an electron multiplying CCD eight times per revolution, we can rebin our data in the time domain after acquisition with little noise penalty, thereby allowing us to explore the polarization evolution of these rapidly variable objects for the first time.
\end{abstract}

Keywords: robotic telescopes, gamma ray bursts, polarimeters, astronomical instrumentation

\section{INTRODUCTION}

The Liverpool Telescope ${ }^{1}$ (LT) is a 2.0 metre fully robotic telescope. It specializes in time variable and rapid reaction astronomy. The telescope Acquisition \& Guidance (A\&G) unit can host up to five instruments ${ }^{2}$ with the beam able to be directed to one of four side ports via a folding mirror, or, with the folding mirror removed from the beam, to a straight through port.

A principle science goal of the Liverpool Telescope is the rapid ( $<3$ minutes) follow up of the optical transient sources associated with Gamma Ray Bursts (GRBs). ${ }^{3}$ This has had considerable success ${ }^{4}$ with multicolour lightcurves of bursts now routinely in production. ${ }^{5}$ In 2006 an area that remained to be explored in early-time GRB studies however was polarimetry. There are good reasons to suspect that at very early times radiation from GRBs may be strongly polarized, and measurements of this would place strong constraints on their geometry and magnetic field strength and structure. With this in mind a novel optical polarimeter (RINGO) with the wide field and high time resolution necessary to observe GRBs was developed and deployed on the Liverpool Telescope in 2006. Since then RINGO has successfully measured the polarization of two bursts: GRB $060418^{6}$ and GRB 090102. ${ }^{7}$ The sensitivity of RINGO is limited however, meaning a long-wait between accessable bursts. In order that we could more rapidly build up a reasonable sample of the polarization properties of GRBs, a new instrument (RINGO2) was therefore developed and deployed to the telescope in 2009. The new instrument has $\sim 10$ times the sensitivity of the orignal RINGO. In this paper we will briefly describe the original instrument in Section 2, explaining where the limitations on its sensitivity arise. In Section 3 we will present the design of RINGO2, explaining how the use of electron-multiplying CCD technology allows us to improve our sensitivity and time resolution. Section 4 will then present the results of our commissioning observations and briefly discuss data reduction for the new instrument. Finally in Section 5 we present our conclusions.

\section{RINGO}

Despite the rapid development in our understanding of the physics of GRBs since the discovery of the first X-ray ${ }^{8}$ and optical ${ }^{9}$ afterglows in 1997, the fundamental emission mechanisms and early time evolution of the ejecta remain unknown. Given that GRBs lie at cosmological distances, they will remain spatially unresolved by all current and future instrumentation. Measurements of the degree and angle of polarization in their early-time light curves could therefore provide a uniquely powerful probe of the geometry of the ejecta and magnetic field strength and structure.

email: ias@astro.livjm.ac.uk 


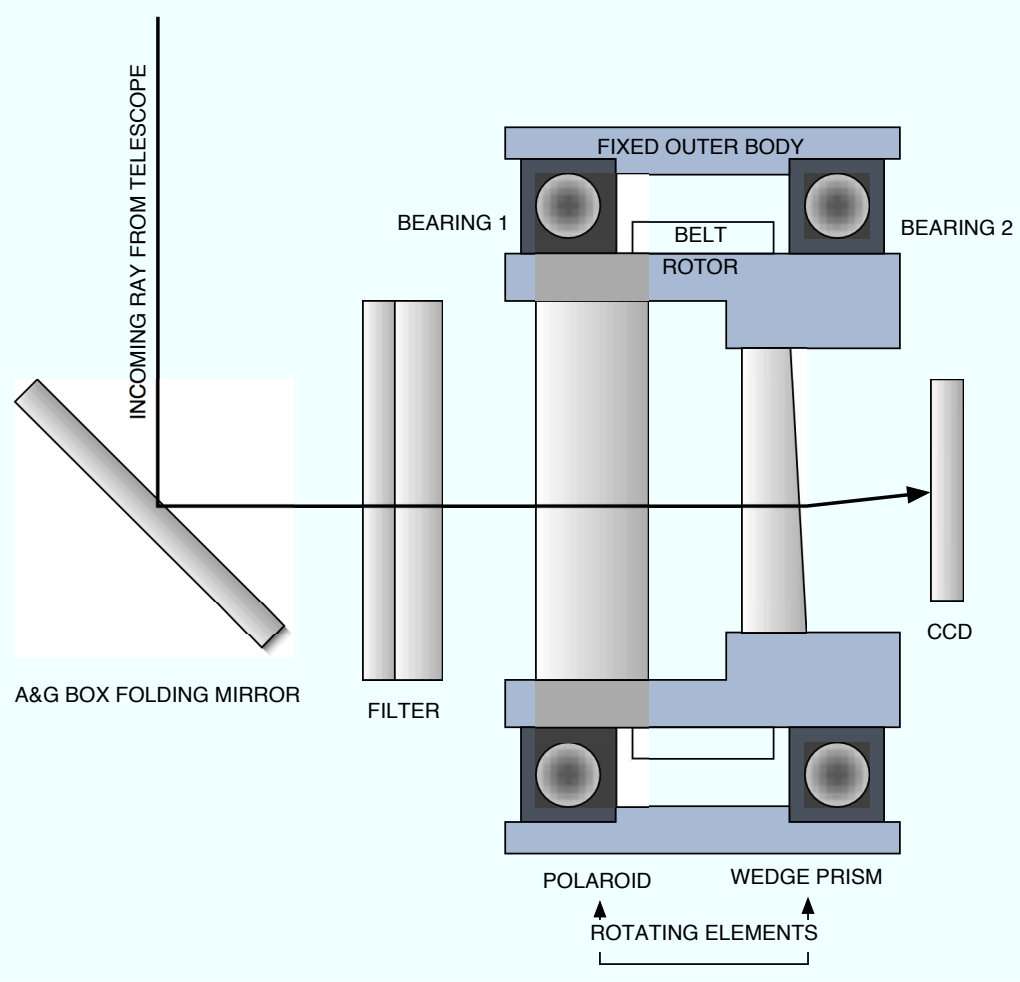

Figure 1. Layout of the original RINGO instrument. The time varying signal from the rotating Polaroid is turned into a spatial modulation using the deviating wedge prism and recorded in a long exposure on the detector. In RINGO2 the wedge prism is removed, and the signal recorded in a series of short exposures on an EMCCD detector.

Long-duration GRBs are understood to be energetic explosions at cosmological distances, caused by the dissipation of a relativistic outflow, or fireball expansion, associated with the gravitational collapse of a massive star to a compact remnant. ${ }^{10}$ Although it is generally accepted that the afterglow emission is synchrotron radiation whose observed properties are broadly explained by a highly-relativistic geometrically-beamed fireball, the driving mechanism of the fireball and, in particular, the role of magnetic fields, is still unknown. It should also be noted that the emission mechanisms for prompt and afterglow optical sources could be quite different.

If GRB ejecta have large scale magnetic fields, then the prompt $\gamma$-ray, X-ray and optical emission and the reverse shock emission should be polarized. ${ }^{11}$ Optical polarization is generally measured via a ratio of fluxes in two or more polarizations. Traditionally this is done by using a modulator/analyzer pair taking separate exposures. However this approach does not work for a rapidly varying object such as a GRB afterglow - a one per-cent "fade" between subsequent exposures implying a false one per-cent polarization signal for example. In 2006 we therefore decided to develop an instrument for the Liverpool Telescope ${ }^{1}$ that can make a "single-shot" polarization measurement. The instrument also required a wide field of view (so that it could respond to SWIFT BAT alerts and be on target during the prompt/reverse shock emission phases). We therefore developed and built the RINGO ${ }^{12}$ polarimeter, which used a fast rotating Polaroid to modulate the incoming beam, followed by corotating deviating optics that transfer each star image into a ring that is recorded on a CCD (Figure 1). Any polarization signal present in the incoming light is mapped out around the ring in a $\sin 2 \theta$ pattern. ${ }^{13,14}$ RINGO has a limiting magnitude of $R \sim 15$ for a polarization measurement with a precision of around $4 \%$.

RINGO was first used in 2006, when it observed GRB 060418 at 203s after the gamma ray burst and coincident with the time of deceleration of the fireball. At this time the reverse (assuming it was present) and forward shock components would have contributed equally to the observed light. For GRB 060418 a $2 \sigma$ upper limit on optical polarization of $\mathrm{P}<8 \%$ was measured in the combined light from the emitting regions. ${ }^{6}$

RINGO was next used in 2009, when it observed GRB 090102. ${ }^{15}$ The steep-shallow decay of optical emission 


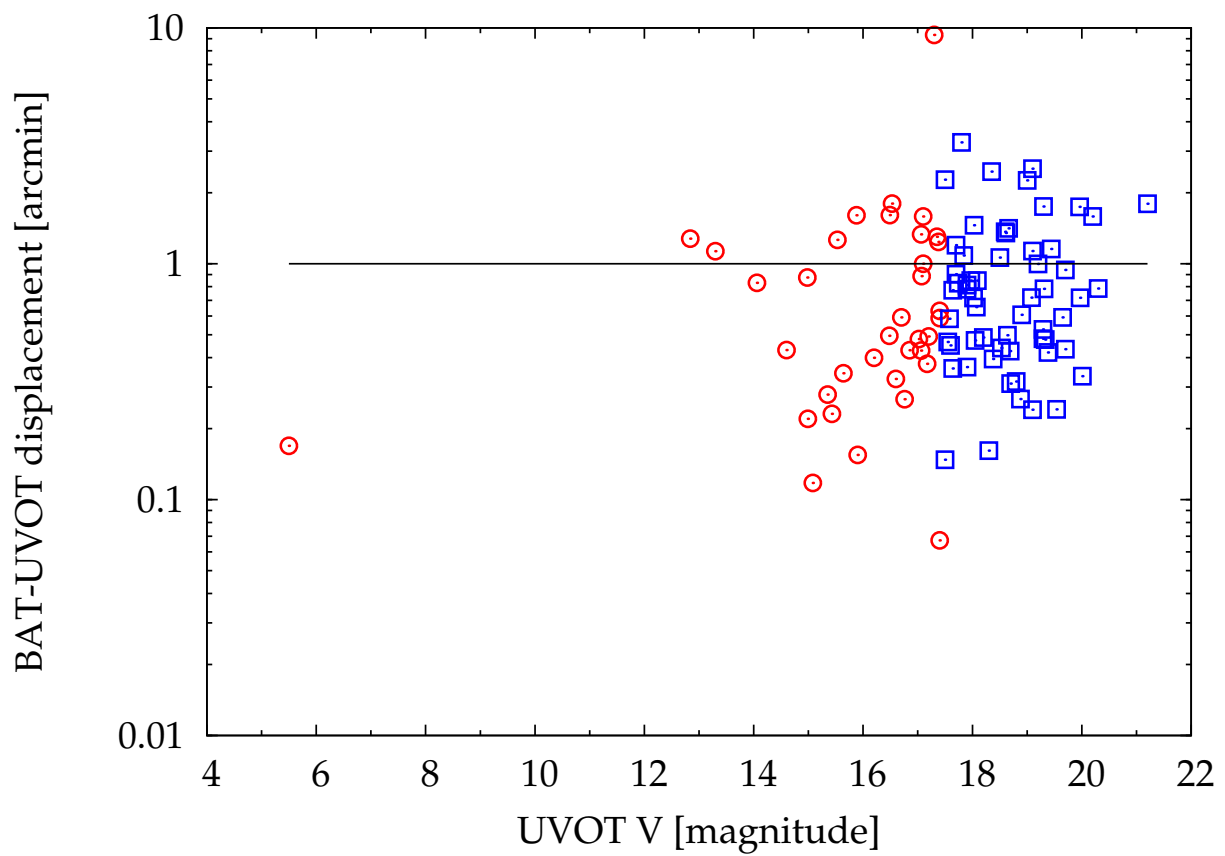

Figure 2. Magnitude distribution of all SWIFT bursts with an optical counterpart until January 2009. The original RINGO instrument had a limiting magnitude of $\sim 15$. RINGO2 improves this to $\sim 17$. Both instruments have a field of view of $4 \times 4$ arcmin.

from GRB 090102 is characteristic of an afterglow whose early-time light is dominated by fading radiation generated in the reverse shock. A single 60-second RINGO exposure was obtained starting 160.8 seconds after the trigger time. RINGO measured the optical $(4600-7200 \AA)$ polarization of GRB 090102 as $P=10.2 \pm 1.3 \%,{ }^{7}$ implying a the presence of large-scale ordered magnetic fields in the relativistic outflow. As the measurement was obtained while the reverse-shock emission was dominant in GRB 090102, the detection of significant polarization provides the first direct evidence that such magnetic fields are present when significant reverse shock emission is produced.

The difference between these two results can be naively understood in terms of the relative strengths of the reverse and forward shocks in the two bursts and possible differences in the magnetic field strengths. However making definitive statements discriminating between models on the basis of only two data points is of course impossible, and what is really required is a good sample of $\sim 10$ bursts.

An analysis of the RINGO, backed up by calculation, ${ }^{12}$ shows the the sensitivity of the instrument is dominated by the night sky brightness. Because the flux from the object is spread over a large ring, then the sky contribution will always dominate over counts from the object itself. RINGO therefore has a limiting magnitude of around $R \sim 15$ to obtain $\sim 4 \%$ polarimetric precision with a 60 second exposure.

In order to improve our sample size we must therefore observe a larger number of bursts. So far we have observed 2 bursts in four years of operation. Figure 2 shows an analysis of all bright SWIFT GRBs with optical counterparts observed up to January 2009. This shows that there were 8 potential bursts observable to the RINGO sensitivity limit over the whole sky during that period. Scaling for sky visibility and hours of darkness the two observations made to data are therefore at the expected rate. It is therefore apparent that in order to obtain our sample of 10 bursts in a reasonable ( $~ 4$ year) timescale we must increase the sensitivity to around $R \sim 17$. This is the primary requirement for the new RINGO2 instrument 


\section{RINGO2}

\subsection{Requirements}

As discussed above, our principle requirement for RINGO2 is a roughly two magnitude sensitivity gain over RINGO, allowing a sample of 10 bursts to be constructed over a 4 year timeframe (Figure 2). Figure 2 also shows that the distribution of Swift $\gamma$-ray position errors implies that a field of view of $\sim 4$ arcmin (as was originally chosen for RINGO for the same reason) remains appropriate if we wish to follow up all bursts detected by the Swift BAT without having to wait for X-ray (XRT) positions as these can sometimes be delayed either by satellite pointing constraints or other reasons.

The LT A\&G box can host up to 5 instruments simultaneously, using a folding mirror to direct the beam to one of four side ports. The time to change between side ports is $\sim 30$ seconds. While in normal use this time is relatively unimportant, with a fast decaying (both in intensity and potential polarization signal) GRB optical transient, this delay can be significant. Given the expected rapid decay of the polarization signal, it was seen as vital that the polarization measurement be obtained as soon as possible after the burst trigger. Therefore the obvious strategy of locating the burst using the telescope CCD imager (RATCam) and then carrying out a reacquisition to place a narrow field polarimeter onto the burst was rejected as adding too much overhead (calculated at $\sim 2$ minutes including the exposure time for the CCD exposures) before making the polarimetric measurement. It was therefore decided that the field of view of the RINGO2 should remain sufficient (i.e. $>4 \times 4$ arcminutes) that this intermediate step was not necessary.

Another important consideration is time resolution. This is important for two reasons. Firstly GRB's themselves vary photometrically on timescales of $\sim$ few seconds. This immediately rules out traditional polarimeters that rely on taking two exposures one after each other after rotating a modulating element and difference the two signals to determine the degree of polarization. The intrinsic photometric variability of the GRB would create a false polarimetric signal. We also must consider the likelihood the the polarization of the GRB may also vary considerably on short timescales. Recent analysis of INTEGRAL date on GRB041219A shows large variability of both the percentage and angle of polarization in gamma-ray emission on short (10 second) timescales, demonstrating that averaging over timescales of $\sim 1$ minute can completely wash out the polarization signal.

The same filter combination as was developed for RINGO was also chosen for RINGO2. In order to derive the optimal wavelength range for that instrument, an analysis of the colours of early GRB light curves was undertaken. This showed they were on average red, with $B-V \sim V-R \sim 1$. A series of simple signal to noise ratio calculations showed that there was no advantage to observing in white light or blueward of the $V$ band in terms of final signal to noise ratio achieved, any additional signal gained being swamped by the additional sky. An additional concern was to avoid fringing on the CCD. Experience with RATCam shows that fringing becomes problematical in the $I$ band. A simple prescription for a " $V+R$ " filter was therefore derived. This consists of 3mm Schott GG475 cemented to $2 \mathrm{~mm}$ KG3. The resulting bandpass is shown in Figure 3.

\subsection{Instrument design and construction}

Our original RINGO instrument was based on the ring polarimeter concept which was revived by Clarke \& Neumayer ${ }^{13}$ based upon the principle proposed by Treanor. ${ }^{14}$ This uses a rotating Polaroid to modulate any polarized flux in the collected radiation and is followed by co-rotating deviating optics so that each star image is recorded on the CCD chip as a circular pattern (Figure 1). The (time-varying) polarization signal is therefore mapped out twice around the circumference of each recorded image as a spatially varying signal. Reference ${ }^{13}$ provides more details of the advantages of such a device, including its potential for high precision measurements using CCD detectors (since it spreads the flux over many pixels around the ring, thereby reducing the normal saturation constraints on high precision CCD photometry). A disadvantage of the design is that in crowded fields the rings can overlap causing problems in extraction (Figure 4). In addition the speed of rotation has an impact on the polarimetric accuracy that can be obtained as any "non-integer" number of rotations with respect to the exposure time will impose a false signal on the ring profile, limiting polarimetric accuracy for bright objects (short exposure times). As already mentioned the principle disadvantage of this design however is caused by the spreading of the object flux around the ring. The minimum ring diameter was set by the atmospheric seeing such that it does not cause flux to "spill" across the ring or between adjacent sections around the ring where 


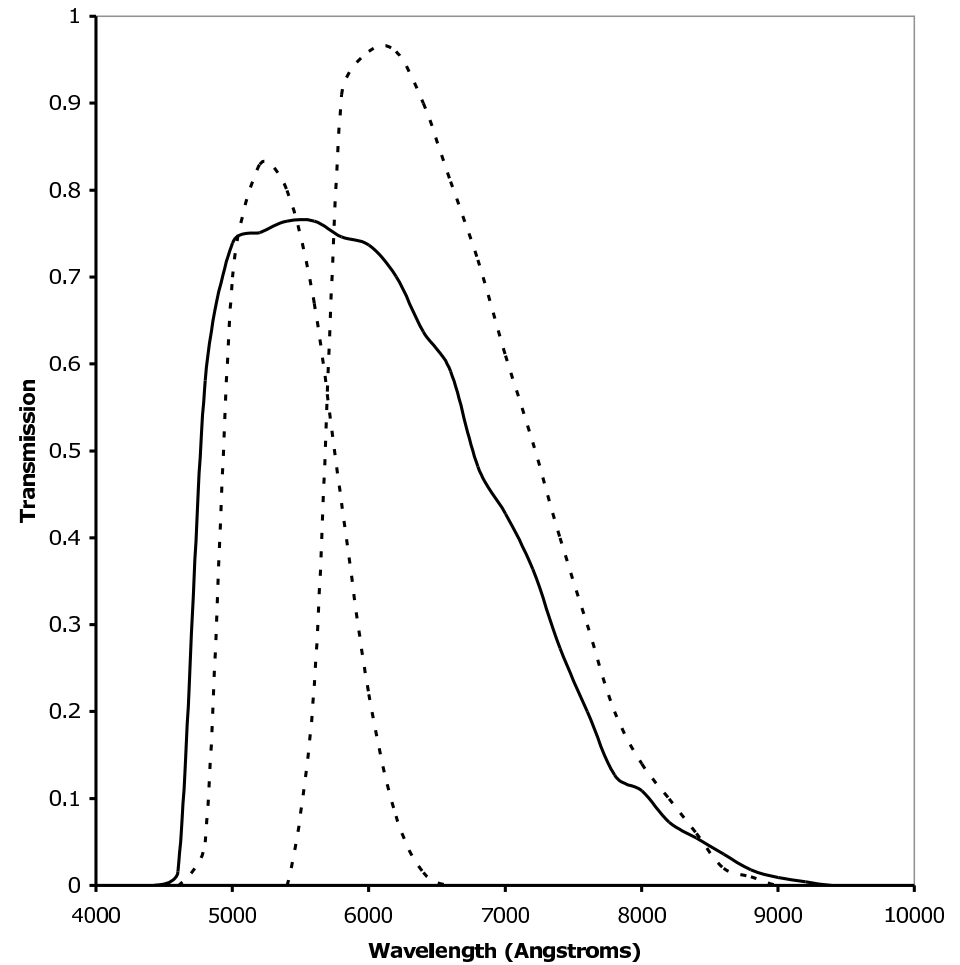

Figure 3. Filter Transmission curve (solid line) for RINGO2 custom filter (3mm GG475 + 2mm KG3) compared to Bessell ${ }^{16} V$ and $R$ curves (dotted lines).

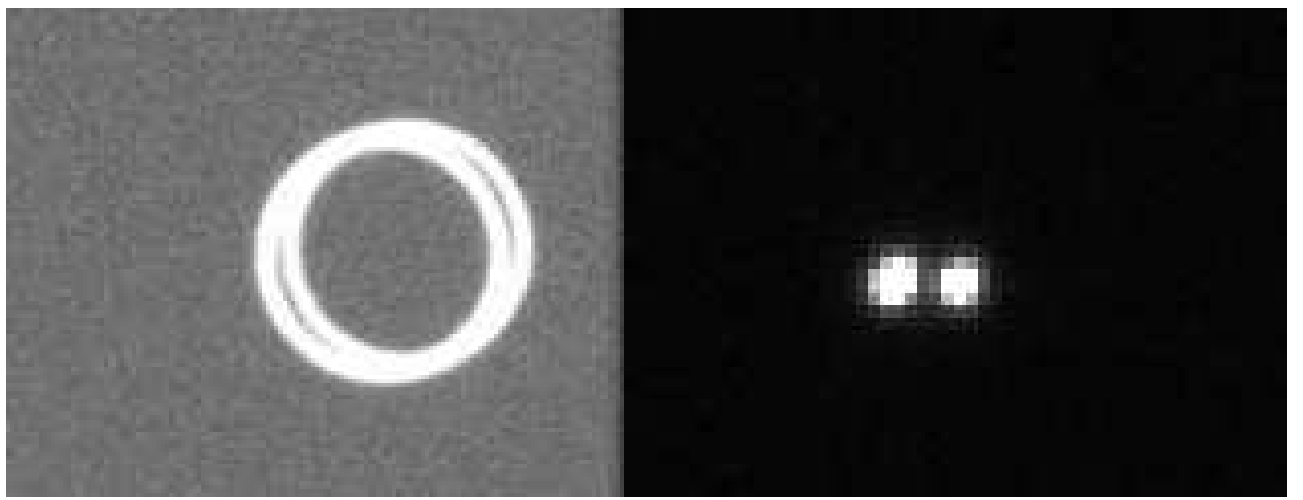

Figure 4. RINGO (left) and RINGO2 (right) observations of the zero-polarization standard double star GD319. The RINGO data are confused by the overlapping rings, a problem which does not affect RINGO2. 


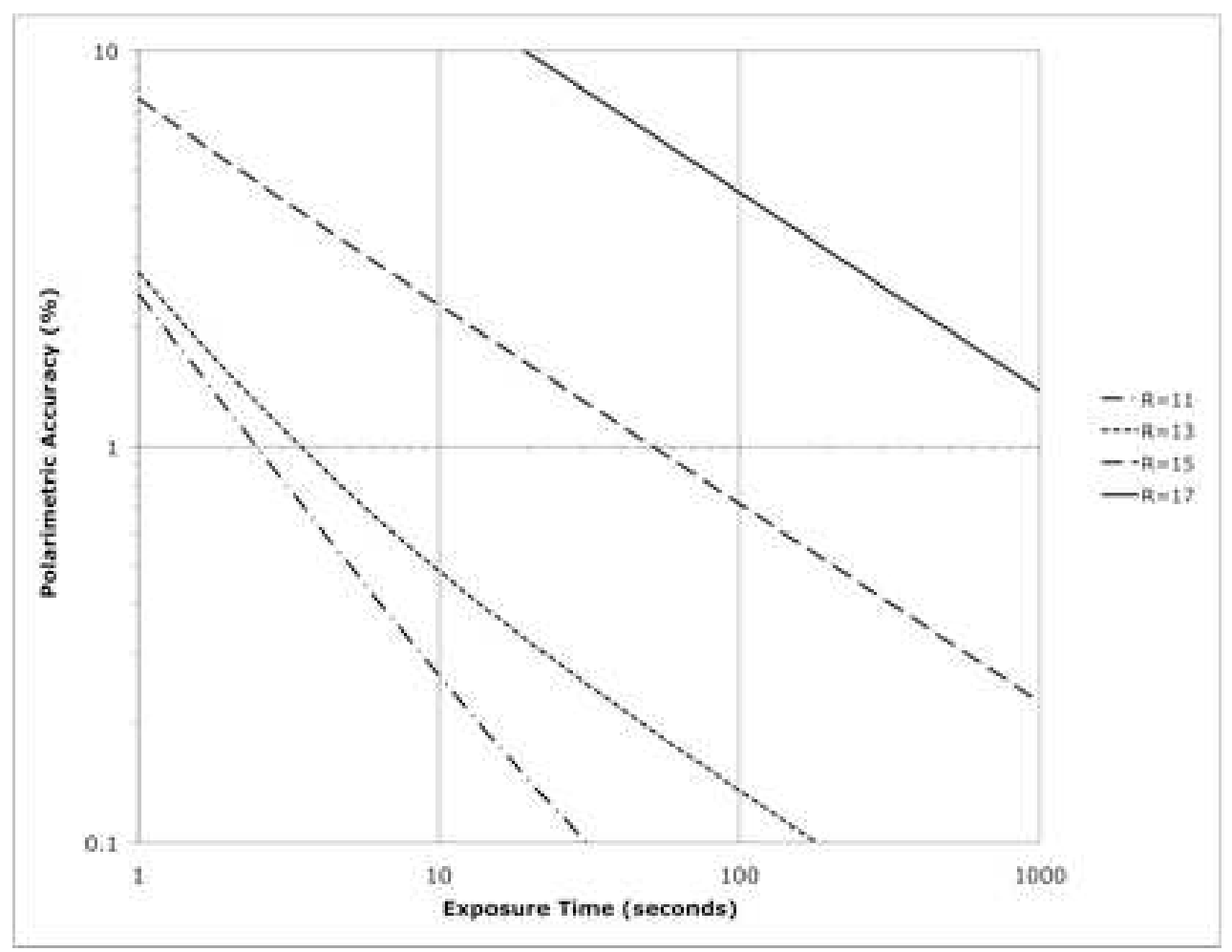

Figure 5. Calculated sensitivity curves for RINGO.

independent measurements of the flux are made in the reduction process. This must be traded off against the increased contribution of sky noise to the measurement that is made when the ring diameter (and hence the area of the ring) is made larger. In our previous paper describing RINGO $^{12}$ we derived equations characterizing these effects. The final polarimetric error of the instrument was then be modelled by adding the rotation and photon-counting components of the error in quadrature and led us to a rotation speed of $500 \mathrm{rpm}$ with a ring diameter of 30 arcsec (Figure 5). Note how for bright objects $(R<12)$ the achieved accuracy is dominated by the rotation speed, whereas for fainter objects the sky noise photon statistics dominate.

For RINGO2 we wished to overcome both of these effects, while also improving our time resolution. The key to achieving this is the recent commercial availability of electron-multiplying CCD cameras. These use the E2V L3CCD detector which incorporate an additional on chip charge multiplication stage between the readout register and the output amplifier of the detector. This uses a process of "impact-ionization" to multiply the signal in a low-noise fashion, allowing very fast readout (up to several hundred frames per second) with negligible $(<1$ ADU) readout noise (with an associated penalty in dynamic range). These detectors have mostly been used in lucky-imaging ${ }^{17}$ applications to date, however they are also ideal for our purpose. By dispensing with the deviating optics in the original RINGO design, the EMCCD can rapidly read out the time varying signal from the polarizer without having to go through the intermediate step of converting it to a spatial signal ("the ring"). By removing the excess sky signal that RINGO suffered from this significantly improves our sensitivity (Figure 6). It also means that (since the read-noise is negligible) the data can be rebinned on any timescale after acquisition. The advantage is obvious for sources such as GRB where the object magnitude is both unknown and rapidly variable. We can trade polarimetric accuracy for time resolution after the event (i.e. during data reduction) in order best optimize the science data products.

The analysis of rotating Polaroid data ${ }^{13}$ requires the signal to be divided into 8 time bins per Polaroid rotation. We therefore chose a rotation period of $\sim 60 \mathrm{rpm}$ for the Polaroid in RINGO2, implying an exposure time of $125 \mathrm{msec}$ for the EMCCD camera (i.e. 8 frames per second).

Our choice of EMCCD camera was driven by a trade-off between sensor size and readout speed. We considered 


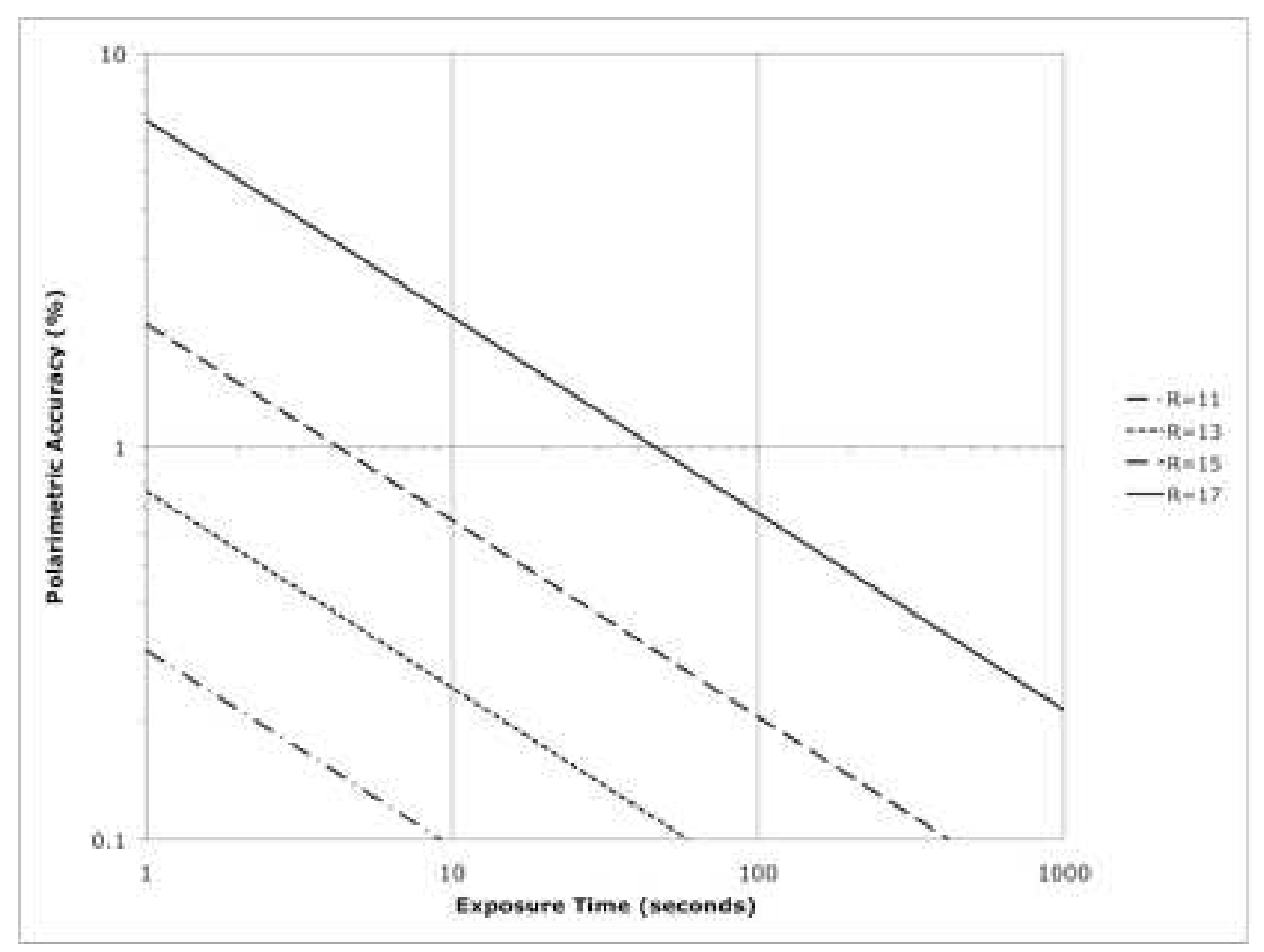

Figure 6. Calculated sensitivity curves for RINGO2. Note the $\sim 2$ magnitude improvement over RINGO.

cameras based around the E2V CCD97 detector which has $512 \times 51216 \mu \mathrm{m}$ pixels and a maximum readout speed of 35 frames per second and the E2V CCD201 which has $1024 \times 1024$ pixels $13 \mu \mathrm{m}$ pixels and a maximum readout speed of 9 frames per second. The CCD97 detector has an image area of $8.2 \times 8.2 \mathrm{~mm}$ and the CCD201 $13.3 \times 13.3 \mathrm{~mm}$. As the Liverpool Telescope pixel scale is $10.1 \mathrm{arcsec} / \mathrm{mm}$, to obtain our desired field of view $(4 \times 4$ arcminutes) this therefore implies that reimaging optics will be required with either detector, with a linear magnification of $\sim 3 \times$ for the CCD97 and $\sim 2 \times$ for the the CCD201. These would yield pixel scales of $\sim 0.4$ arcsec for the CCD97 and $\sim 0.25$ arcsec for the CCD201. Given the telescope median seeing of $\sim 1$ arcsec either of these options is acceptable. We therefore chose the smaller (and hence faster reading) CCD97 detector, as we were concerned that our requirement of 8 frames per second readout was close to the maximum recommended for the CCD201. The CCD97 is available in a Peltier cooled prepackaged camera from Andor as their model iXon +897 . This was therefore purchased and is operated air cooled at $-60^{\circ} \mathrm{C}$. The camera uses a PCI interface card and was provided with a Linux software development kit (SDK) which we used for software development.

Photographic lenses were used as collimator-camera pair to provide the reimaging optics. The collimator lens used is a medium format Mamiya $150 \mathrm{~mm} \mathrm{f} / 3.5 \mathrm{~N}$. This has sufficient image circle to fully encompass the beam from the 4x4 arcmin field at telescope focal plane. The camera lens is a $35 \mathrm{~mm}$ format Nikon AF-D 50mm f/1.4.

The instrument housing, polarizer (Meadowlark) and polarization rotation mechanism were reused from RINGO. The rotor is housed within two ball bearings. A rubber drive belt makes a friction contact with the rotor to provide a clutch action on start up. The use of a belt drive also isolates the rotor from any vibrations associated with the motor, which is compliantly mounted onto the instrument. The motor is a brushless (to reduce electromagnetic interference during CCD readout) Creuzet high torque $24 \mathrm{~V}$ DC model with integral gearbox. In combination with the belt drive ratio this gives the required final speed at the rotor of $\sim 60 \mathrm{rpm}$. In operation the motor is left running continually (day and night) so that no delays for system spin-up are encountered on a GRB trigger.

The polarization measurement method employed requires that exactly 8 measurements be taken per single rotation of the Polaroid. In addition the measurements should start at the same rotation angle each time. As 


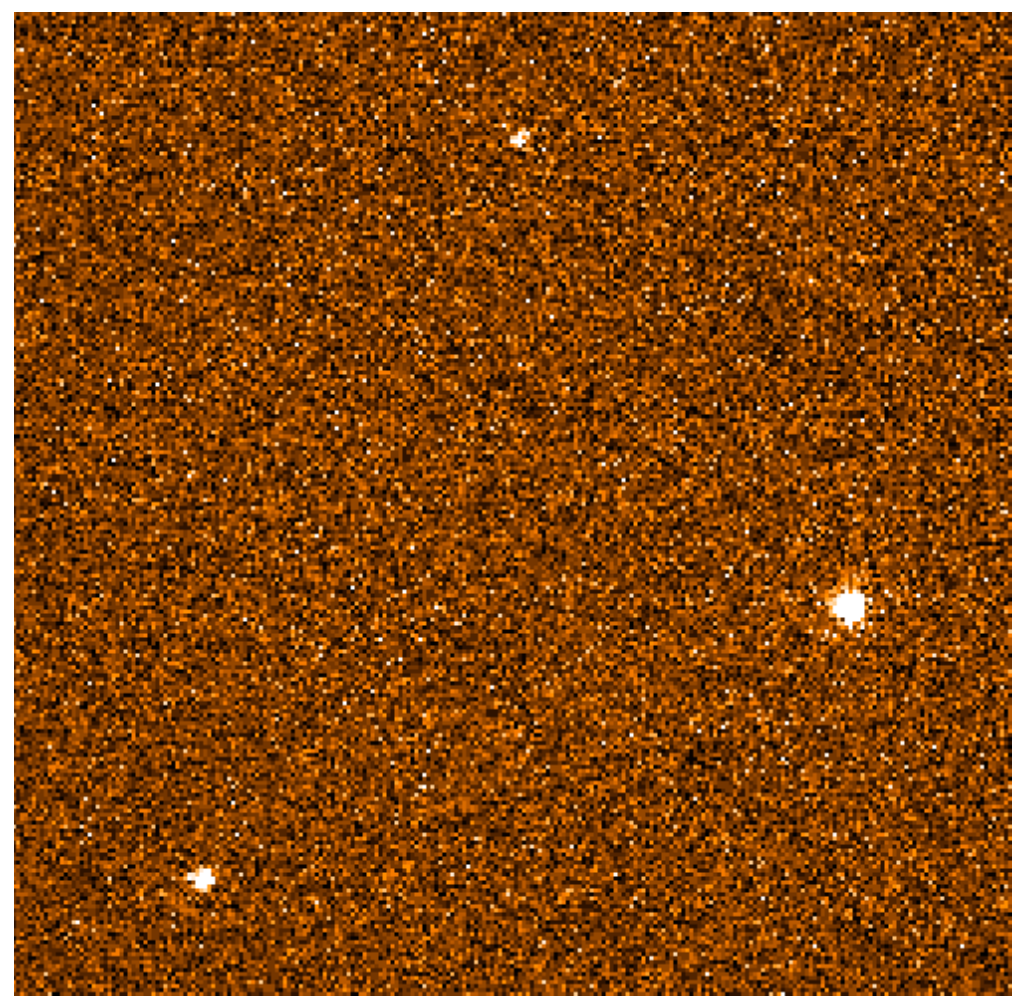

Figure 7. Single 125 msec RINGO2 exposure of the Landolt standard field around PG0231. The faint object at the top of the frame has $V \sim 16$ and would be invisible in a 10 second RINGO exposure, demonstrating the improved sensitivity of the instrument.

the polaroid mechanism is allowed to rotate freely this implies that the camera exposures must be triggered by the position of the rotor. This is accomplished by inserting small metal plips into the rotor. These are located equally spaced around the circumference and read by a high speed proximity sensor. An additional "home" plip is located on the rotors to provide a zero rotation reference mark. These are combined along with an output trigger signal from the camera (indicating that the camera is ready to begin a sequence of exposures) in custombuilt logic circuitry in order to provide an sequence of input trigger signals to the camera, always beginning at the same rotation angle. The camera is operated in its "external trigger" mode where the start and length of the exposures is determined by the signal level on that input (rather than software). The images from the CCD are automatically read into a circular buffer by the camera device driver. The buffer is then read out by our own instrument software and streamed to disk with added FITS headers.

\section{COMMISSIONING AND DATA REDUCTION}

In theory the reduction of data from the instrument should be relatively straightforward. Following the standard CCD data reductions (dark subtraction, flat-fielding) then a simple photometry package can be used to measure the image intensity in the 8 images associated either with a single full rotation of the polaroid or with coadded sets of 8 images. Plotting the counts from an object for these 8 images taken at different Polaroid angles then reveals the expected $\sin 2 \theta$ modulation (Figure 8 ) and which we term a "polarization trace". The values of the counts from each image are then fitted to the equations given in Reference ${ }^{13}$ which allow the derivation of the Stokes parameters.

In practice the reduction of data is complicated by a number of factors. The beam is reflected off a $45^{\circ}$ folding mirror before entering the instrument. This leads to a significant $(\sim 2 \%)$ additional polarization signal being superimposed on the beam. Additionally there will also be some instrumental depolarization of the original source caused by the telescope and instrument optics. Fortunately an acceptable reduction and calibration of 


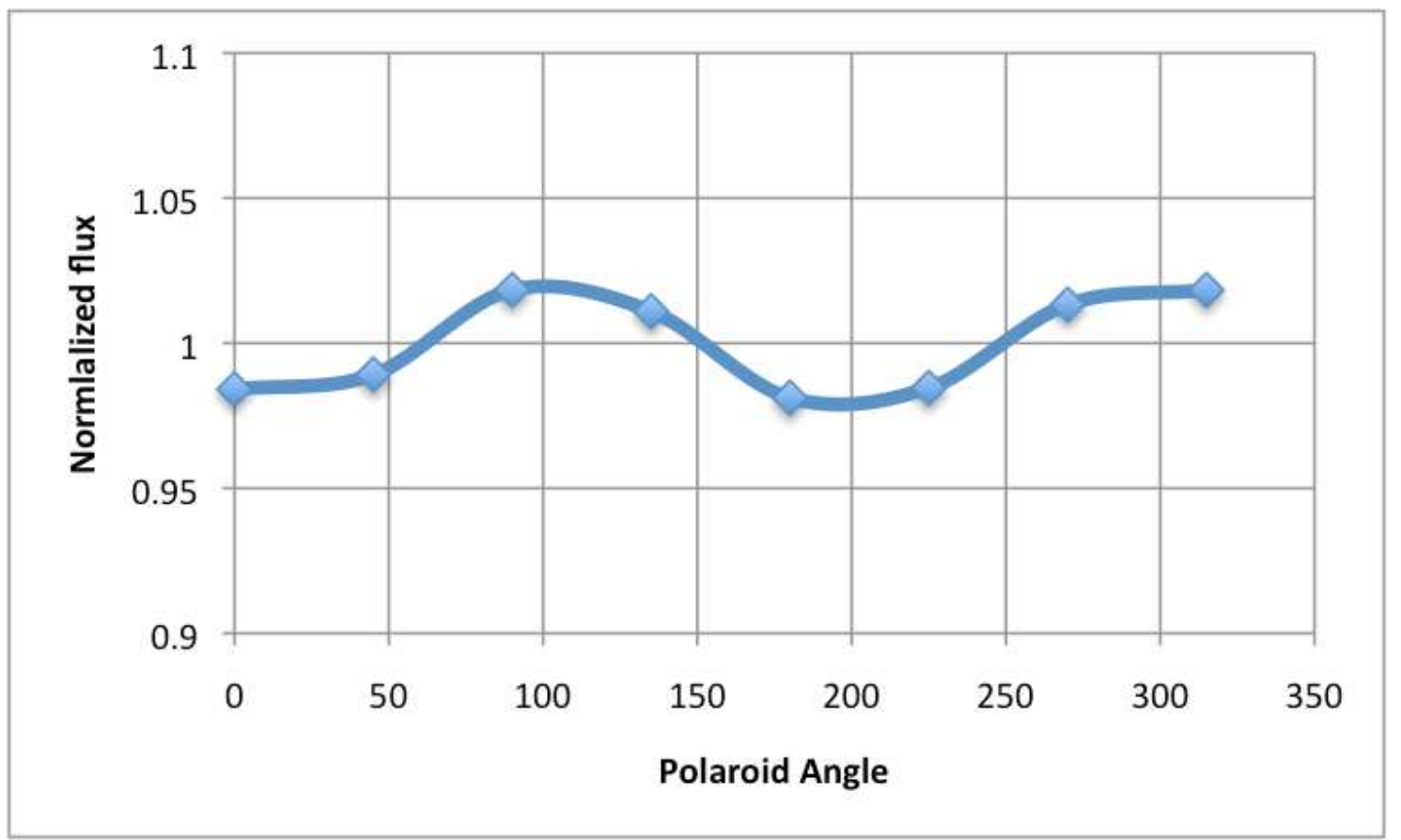

Figure 8. Sky subtracted, normalized trace from the zero-polarized standard G191B2. The residual polarization from the instrument optics can be seen. By dividing this trace into that from the science objects, it can be removed.

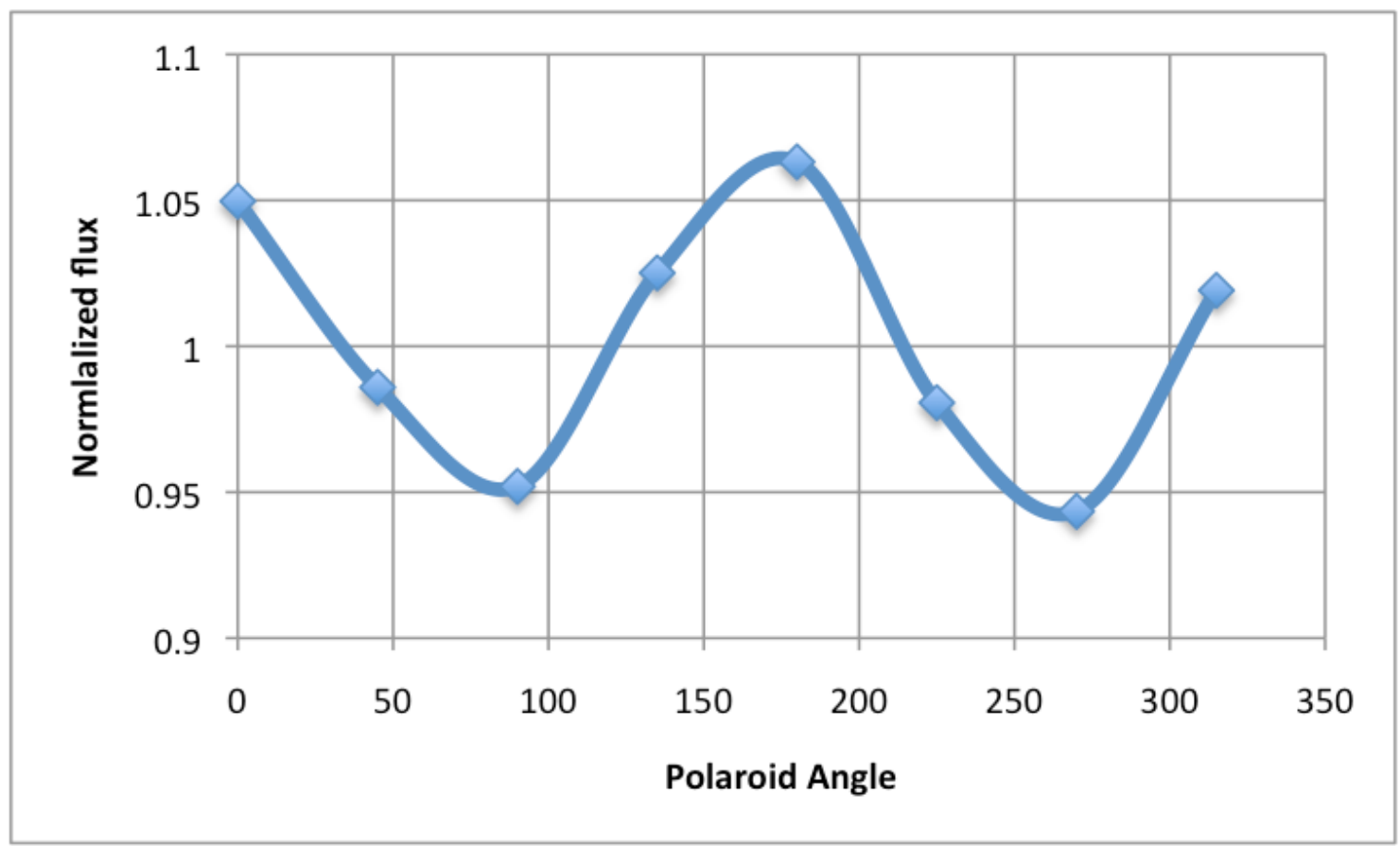

Figure 9. The polarized standard NGC2024-1 before division by the zero-polarization standard. 


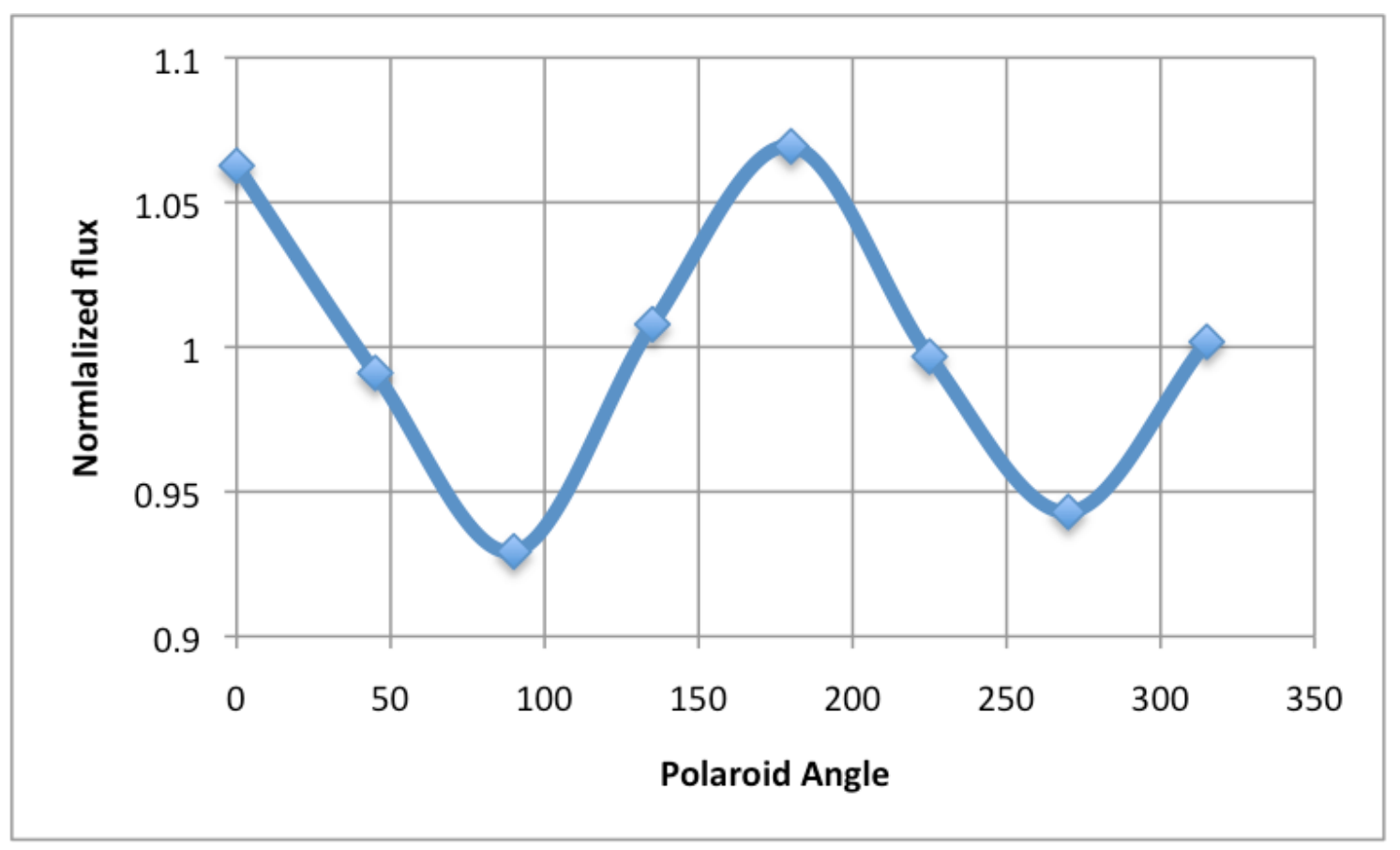

Figure 10. The polarized standard NGC2024-1 with the instrumental polarization signature removed.

the data can be done using observations of polarized standards ${ }^{18}$ both of known zero and non-zero polarization. The zero-polarization standards characterize the effects of instrumental polarization (and also any non uniform rotation of the rotor) in much the same way as a flat-field does for instrumental photometric effects. This zeropolarization trace is therefore divided into the traces of other observations in order to remove these effects. An example zero-polarization standard trace is shown in Figure 8.

In Figure 9 we show a trace of a sample non-zero polarimetric standard. Dividing the zero-polarization trace from Figure 8 into the standard then yields Figure 10. This trace with the instrumental signature removed can then be fitted ${ }^{13}$ and the Stokes parameters derived, free from the effects of instrumental polarization. The derived polarization angle and degree of polarization can then be compared to the published standard values. This then allows the degree of instrumental depolarization to be derived which can then be applied as a correction factor to the science frames.

RINGO2 was built over the summer of 2009 and deployed to the Liverpool Telescope in November 2009. During commissioning a large number of polarimetric standard stars were observed, with repeated measurements made and testing done with the standard located in different parts of the field. An example image taken on the commissioning run and demonstrating the sensitivity of the instrument as compared to RINGO2 can be seen in Figure 7. The instrument stability (determined by ratioing various zero-polarization standards and repeated observation of polarized standards) was determined in commissioning to be better than $0.2 \%$.

\section{CONCLUSION}

The RINGO2 instrument was developed and deployed in under 6 months and for a budget of $£ 30,000(\sim \$ 50,000)$, the majority of which was the cost of the CCD camera. Its novel design gives it a wide field of view and the fast response necessary for measuring the polarization of gamma ray burst optical transients. Commissioning tests have shown that it can make repeatable measurements to a precision of $0.2 \%$. It is now in routine operation on the Liverpool Telescope for Gamma Ray Burst followup and other science programmes requiring regular monitoring of variable polarization sources (for example the nucleus and jets of the AGN M87). 


\section{ACKNOWLEDGMENTS}

The Liverpool Telescope is located at the Observatorio del Roque do Los Muchachos, La Palma, Canary Islands, Spain. It was designed and built by Telescope Technologies Ltd and is owned and operated by Liverpool John Moores University with financial support from the UK Science and Technology Facilities Council.

The hardware costs of RINGO2 were supported using funding from the UK Higher Eduction Funding Council RCIF project.

\section{REFERENCES}

[1] Steele, I. A., Smith, R. J., Rees, P. C., Baker, I. P., Bates, S. D., Bode, M. F., Bowman, M. K., Carter, D., Etherton, J., Ford, M. J., Fraser, S. N., Gomboc, A., Lett, R. D. J., Mansfield, A. G., Marchant, J. M., Medrano-Cerda, G. A., Mottram, C. J., Raback, D., Scott, A. B., Tomlinson, M. D., and Zamanov, R., "The Liverpool Telescope: performance and first results," in [Proceedings of the SPIE, Volume 5489, pp. 679-692 (2004).], Oschmann, J. M., ed., 679-692 (Oct. 2004).

[2] Mottram, C. J., Steele, I. A., and Morales, L., "Design of low cost and reliable instrumentation for robotic telescopes," in [Ground-based Instrumentation for Astronomy. Edited by Alan F. M. Moorwood and Iye Masanori. Proceedings of the SPIE, Volume 5492, pp. 677-688 (2004).], Moorwood, A. F. M. and Iye, M., eds., 677-688 (Sept. 2004).

[3] Guidorzi, C., Monfardini, A., Gomboc, A., Mottram, C. J., Mundell, C. G., Steele, I. A., Carter, D., Bode, M. F., Smith, R. J., Fraser, S. N., Burgdorf, M. J., and Newsam, A. M., "The Automatic Real-Time Gamma-Ray Burst Pipeline of the 2 m Liverpool Telescope," PASP 118, 288-296 (Feb. 2006).

[4] Guidorzi, C., Monfardini, A., Gomboc, A., Mundell, C. G., Steele, I. A., Carter, D., Bode, M. F., Smith, R. J., Mottram, C. J., Burgdorf, M. J., Tanvir, N. R., Masetti, N., and Pian, E., "The Early Multicolor Afterglow of GRB 050502a: Possible Evidence for a Uniform Medium with Density Clumps," ApJL 630, L121-L124 (Sept. 2005).

[5] Melandri, A., Mundell, C. G., Kobayashi, S., Guidorzi, C., Gomboc, A., Steele, I. A., Smith, R. J., Bersier, D., Mottram, C. J., Carter, D., Bode, M. F., O’Brien, P. T., Tanvir, N. R., Rol, E., and Chapman, R., "The Early-Time Optical Properties of Gamma-Ray Burst Afterglows," ApJ 686, 1209-1230 (Oct. 2008).

[6] Mundell, C. G., Steele, I. A., Smith, R. J., Kobayashi, S., Melandri, A., Guidorzi, C., Gomboc, A., Mottram, C. J., Clarke, D., Monfardini, A., Carter, D., and Bersier, D., "Early Optical Polarization of a Gamma-Ray Burst Afterglow," Science 315, 1822- (Mar. 2007).

[7] Steele, I. A., Mundell, C. G., Smith, R. J., Kobayashi, S., and Guidorzi, C., "Ten per cent polarized optical emission from GRB090102," Nature 462, 767-769 (Dec. 2009).

[8] Costa, E., Frontera, F., Heise, J., Feroci, M., in 't Zand, J., Fiore, F., Cinti, M. N., dal Fiume, D., Nicastro, L., Orlandini, M., Palazzi, E., Rapisarda, M., Zavattini, G., Jager, R., Parmar, A., Owens, A., Molendi, S., Cusumano, G., Maccarone, M. C., Giarrusso, S., Coletta, A., Antonelli, L. A., Giommi, P., Muller, J. M., Piro, L., and Butler, R. C., "Discovery of an X-ray afterglow associated with the gamma-ray burst of 28 February 1997.," Nature 387, 783-785 (June 1997).

[9] van Paradijs, J., Groot, P. J., Galama, T., Kouveliotou, C., Strom, R. G., Telting, J., Rutten, R. G. M., Fishman, G. J., Meegan, C. A., Pettini, M., Tanvir, N., Bloom, J., Pedersen, H., Nordgaard-Nielsen, H. U., Linden-Vornle, M., Melnick, J., van der Steene, G., Bremer, M., Naber, R., Heise, J., in 't Zand, J., Costa, E., Feroci, M., Piro, L., Frontera, F., Zavattini, G., Nicastro, L., Palazzi, E., Bennet, K., Hanlon, L., and Parmar, A., "Transient optical emission from the error box of the gamma-ray burst of 28 February 1997.," Nature 386, 686-689 (1997).

[10] Piran, T., "The physics of gamma-ray bursts," Reviews of Modern Physics 76, 1143-1210 (Jan. 2005).

[11] Lyutikov, M., "The electromagnetic model of gamma-ray bursts," New Journal of Physics 8, 119-+ (July 2006).

[12] Steele, I. A., Bates, S. D., Carter, D., Clarke, D., Gomboc, A., Guidorzi, C., Melandri, A., Monfardini, A., Mottram, C. J., Mundell, C. G., Scott, A. B., Smith, R. J., and Swindlehurst, J., "RINGO: a novel ring polarimeter for rapid GRB followup," in [Society of Photo-Optical Instrumentation Engineers (SPIE) Conference Series], Society of Photo-Optical Instrumentation Engineers (SPIE) Conference Series 6269 (July 2006). 
[13] Clarke, D. and Neumayer, D., "Experiments with a novel CCD stellar polarimeter," $A \mathscr{E} A$ A 383, 360-366 (Jan. 2002).

[14] Treanor, P. F., "A new technique for stellar polarimetry," MNRAS 138, 325-+ (1968).

[15] Gendre, B., Klotz, A., Palazzi, E., Krühler, T., Covino, S., Afonso, P., Antonelli, L. A., Atteia, J. L., D'Avanzo, P., Boër, M., Greiner, J., and Klose, S., "Testing gamma-ray burst models with the afterglow of GRB 090102," MNRAS , 576-+ (Apr. 2010).

[16] Bessell, M. S., "UBVRI passbands," PASP 102, 1181-1199 (Oct. 1990).

[17] Basden, A., Tubbs, B., and Mackay, C., "L3CCD's: Low Readout Noise CCDS in Astronomy," in [Scientific Detectors for Astronomy, The Beginning of a New Era], P. Amico, J. W. Beletic, \& J. E. Belectic, ed., Astrophysics and Space Science Library 300, 599-602 (2004).

[18] Schmidt, G. D., Elston, R., and Lupie, O. L., "The Hubble Space Telescope Northern-Hemisphere grid of stellar polarimetric standards," AJ 104, 1563-1567 (Oct. 1992). 